\title{
A Comparative Systematic Review of COVID-19 and Influenza
}

\author{
Molka Osman ${ }^{1}\left(\mathbb{D}\right.$, Timothée Klopfenstein ${ }^{2}$, Nabil Belfeki ${ }^{3}$, Vincent Gendrin ${ }^{2, *}$ (D) and Souheil Zayet ${ }^{2, *}$ (D) \\ 1 Faculty of Medicine of Tunis, University Tunis El Manar, Tunis 1007, Tunisia; molkaosman@gmail.com \\ 2 Infectious Disease Department, Nord Franche-Comté Hospital, 90400 Trévenans, France; \\ timothee.klopfenstein@hnfc.fr \\ 3 Internal Medicine Department, Groupe Hospitalier Sud Ile de France, 77000 Melun, France; \\ nabil.belfeki@ghsif.fr \\ * Correspondence: vincent.gendrin@hnfc.fr (V.G.); souhail.zayet@gmail.com (S.Z.)
}

Citation: Osman, M.; Klopfenstein

T.; Belfeki, N.; Gendrin, V.; Zayet, S. A Comparative Systematic Review of COVID-19 and Influenza. Viruses 2021, 13, 452. https://doi.org/ $10.3390 /$ v13030452

Academic Editor:

Luis Martinez-Sobrido

Received: 14 February 2021

Accepted: 8 March 2021

Published: 10 March 2021

Publisher's Note: MDPI stays neutral with regard to jurisdictional claims in published maps and institutional affiliations.

Copyright: (c) 2021 by the authors. Licensee MDPI, Basel, Switzerland. This article is an open access article distributed under the terms and conditions of the Creative Commons Attribution (CC BY) license (https:// creativecommons.org/licenses/by/ $4.0 /)$.

\begin{abstract}
Background: Both SARS-CoV-2 and influenza virus share similarities such as clinical features and outcome, laboratory, and radiological findings. Methods: Literature search was done using PubMed to find MEDLINE indexed articles relevant to this study. As of 25 November 2020, the search has been conducted by combining the MeSH words "COVID-19" and "Influenza". Results: Eighteen articles were finally selected in adult patients. Comorbidities such as cardiovascular diseases, diabetes, and obesity were significantly higher in COVID-19 patients, while pulmonary diseases and immunocompromised conditions were significantly more common in influenza patients. The incidence rates of fever, vomiting, ocular and otorhinolaryngological symptoms were found to be significantly higher in influenza patients when compared with COVID-19 patients. However, neurologic symptoms and diarrhea were statistically more frequent in COVID-19 patients. The level of white cell count and procalcitonin was significantly higher in influenza patients, whereas thrombopenia and elevated transaminases were significantly more common in COVID-19 patients. Ground-grass opacities, interlobular septal thickening, and a peripheral distribution were more common in COVID-19 patients than in influenza patients where consolidations and linear opacities were described instead. COVID-19 patients were significantly more often transferred to intensive care unit with a higher rate of mortality. Conclusions: This study estimated differences of COVID-19 and influenza patients which can help clinicians during the co-circulation of the two viruses.
\end{abstract}

Keywords: influenza; COVID-19; SARS-CoV-2; clinical features; laboratory; imaging; systematic review

\section{Introduction}

Coronavirus disease 2019 (COVID-19), an infection caused by severe acute respiratory syndrome coronavirus-2 (SARS-CoV-2), was first reported in December 2019, in Wuhan, province of Hubei in China. Since then, it has evolved into a worldwide pandemic and became one of the world's toughest health problems [1]. Both SARS-CoV-2 and influenza virus share similarities such as viral shedding, route of transmission, and clinical presentation [2]. Influenza affects $5-10 \%$ of adults annually with most cases occurring during the winter season [3]. According to the World Health Organization (WHO), influenza's epidemics are estimated to result in about 3 to 5 million cases of severe illness, and about 290,000 to 650,000 respiratory deaths [4].

There have been few studies comparing COVID-19 with influenza mainly focused on epidemiological features in the general population [5]. However, no review study compared clinical, biological, and radiological characteristics between COVID-19 and influenza. With projections estimating the COVID-19 pandemic to last for another year [2] and it being the influenza season, it is crucial to evaluate the differences between the two diseases. The current study performed a systematic review to compare the clinical features and outcome, laboratory, and radiological findings of COVID-19 patients with influenza adult patients. 


\section{Methods}

This study is based on a review of published literature comparing COVID-19 and influenza. Literature search was done using PubMed to find MEDLINE indexed articles relevant to this study. As of 25 November 2020, the search has been conducted by combining the MeSH words "COVID-19" and "Influenza". At first, citations that were not in English or in French and not a free full text were excluded. Then, we screened citations based on titles and abstracts. Those with irrelevant information or subjects, studies with focus other than comparing COVID-19 and influenza, studies with a population under 18 years old and case reports about coinfection between COVID-19 and influenza were excluded. A $p$-value $<0.05$ was considered significant to compare the two viruses.

\section{Results}

\subsection{Included Citations in the COVID-19 vs. Influenza Review}

On PubMed, 331 articles were initially found, out of which 46 articles were selected. In the end, 18 articles were selected [3,5-21] (8 retrospective studies, 6 cohort studies, 3 casecontrol studies, 1 prospective study, 1 cross-sectional study, 1 review and meta-analysis, and 1 review) (Figure 1 and Table 1)

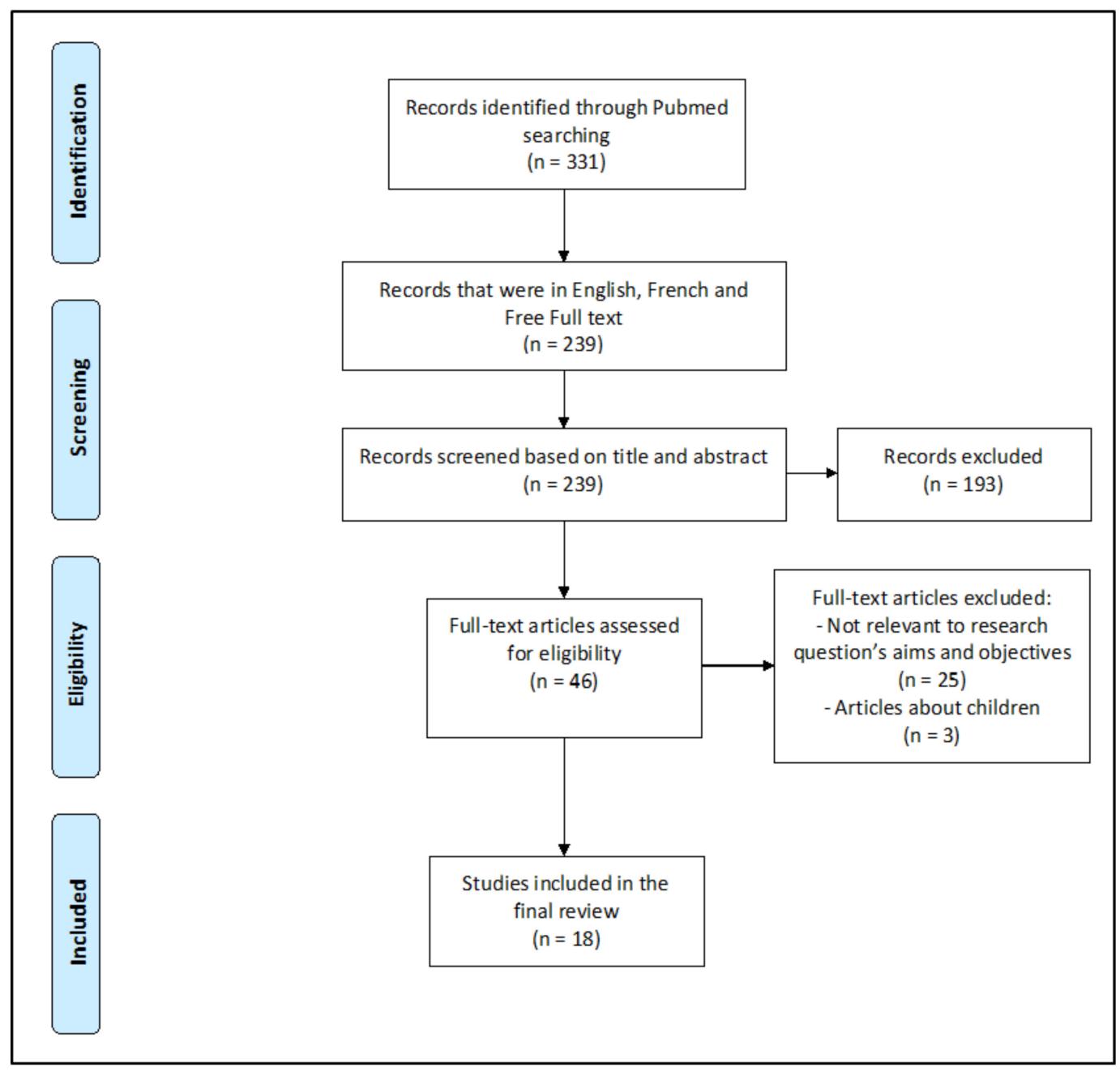

Figure 1. PRISMA guideline flowchart detailing article selection process. 
Table 1. General summary of included citations.

\begin{tabular}{|c|c|c|c|}
\hline References & Country & Study Type ** & $\begin{array}{l}\text { Number of Patients/Studies * } \\
\text { (COVID-19 vs. Influenza) }\end{array}$ \\
\hline Jordan Cates et al. [6] & United States & Cohort & 9401 (3948 vs. 5453 ) \\
\hline Ying Luo et al. [7] & China (Hubei) & Cohort & 2167 (1027 vs. 1140) \\
\hline Jiangnan Chen et al. [8] & China (Shaoxing) & Case-Control & 380 (169 vs. $131 ; 80$ healthy controls) \\
\hline Jiajia Qu et al. [9] & China & Retrospective Cohort & 366 (246 vs. 120$)$ \\
\hline Jianguo Zhang et al. [10] & China & Retrospective cohort & 326 (211 vs. 115$)$ \\
\hline Helene Faury et al. [11] & France (Paris) & Retrospective & 200 (100 vs. 100) \\
\hline Pengfei Li et al. * [5] & - & $\begin{array}{c}\text { Systematic review and } \\
\text { Meta-analysis }\end{array}$ & 197 (113 vs. 84) \\
\hline Mengqi Liu et al. [12] & China (Chongqing) & Retrospective & 180 (122 vs. 48$)$ \\
\hline Xiao Tang et al. [13] & China (Wuhan) & Retrospective case-control & 148 (73 vs. 75$)$ \\
\hline Natalie L. Cobb et al. [14] & United States (Washington) & Retrospective Cohort & 139 (65 vs. 74$)$ \\
\hline Souheil Zayet et al. [15] & France & Retrospective & 124 (70 vs. 54$)$ \\
\hline Hao Wang et al. [16] & China & Retrospective & 105 (13 vs. 92) \\
\hline Liaoyi Lin et al. [17] & China (Wenzhou) & Retrospective & 97 (52 vs. 45$)$ \\
\hline Raija Auvinen et al. [18] & Finland & Prospective study & 61 (28 vs. 33$)$ \\
\hline Zhilan Yin et al. [19] & China & Retrospective & 60 (30 vs. 30$)$ \\
\hline Yi-Hua Lin et al. [20] & China (Xiamen) & $\begin{array}{l}\text { A cross-sectional } \\
\text { retrospective study }\end{array}$ & 57 (35 vs. 22$)$ \\
\hline Jaehee Lee et al. [21] & South Korea (Daegu) & Retrospective & 29 (20 vs. 09$)$ \\
\hline Stephen O. Onigbinde et al. * [3] & - & Review & 17 (09 vs. 08) \\
\hline
\end{tabular}

* Studies for Review or Meta-analysis. ${ }^{* *}$ Cohort studies are used to investigate causes of disease and establish association between risk factors and health outcomes. An outcome-free study population is first identified by the exposure/event of interest and followed in time until the outcome of interest happens. They can be prospective (carried out from the present time into the future) or retrospective (carried out at the present time and look to the past to examine medical events or outcome). Case-Control studies first identify subjects by outcome status (cases), then select from the same source population, subjects without the outcome (control). Cross-sectional study or prevalence study is an observational study that collects data on the subjects of interest at a specific point in time.

\subsection{Studies in Patients with COVID-19 and Influenza}

\subsubsection{Demographic and Clinical Findings}

In this review, 14 studies of 18 [5,6,9-16,18-21] reported a comparison between COVID19 and influenza patients based on demographic findings, comorbidities, clinical features, and outcome (Table 2). Fever and respiratory symptoms such as cough, expectoration or sputum production and dyspnea were the main symptoms in both groups with COVID-19 and influenza; however, they were significantly more frequent in patients with influenza $[5,9-11,13,15,16,19,20]$. Moreover, vomiting, otorhinolaryngological symptoms such as nasal congestion, rhinorrhea, sore throat and ocular symptoms such as tearing and conjunctival hyperhemia were statistically more frequent in patients with influenza than COVID-19 adult patients [5,10-12,15]. In patients infected with COVID-19, the most significant and frequent clinical features were fatigue, neurologic symptoms such as headache (especially facial headache: retro-orbital or frontal headache), anosmia and dysgeusia, gastro-intestinal (GI) symptoms such as diarrhea and acute respiratory distress syndrome (ARDS), compared to influenza patients [11,13-15,18]. In a retrospective case-control study, Tang et al. reported that patients with influenza were more disposed to have productive cough and higher Sequential Organ Failure Assessment (SOFA) scores $(\mathrm{OR}=9.58,(95 \% \mathrm{CI}=1.73-64.72), p=0.011$ and $\mathrm{OR}=2.26(1.12-3.57), p=0.006$, respectively). Meanwhile, COVID-19 patients exhibit symptoms of fatigue or GI symptoms $(\mathrm{OR}=0.91(0.84-0.98), p=0.011 ; \mathrm{OR}=0.12(0.02-0.94), p=0.013$ and $\mathrm{OR}=0.10,(0.01-0.98)$, $p=0.044$, respectively) [13]. 
Table 2. Significant demographic and baseline characteristics, clinical features, and outcome in COVID-19 and influenza adult groups.

\begin{tabular}{|c|c|c|c|c|}
\hline References & Significant Clinical Features/Outcome & COVID-19 (\%) & Influenza $(\%)$ & $p$-Value $<0.05$ \\
\hline \multirow{3}{*}{ Jordan Cates et al. [6] } & Admitted to ICU & 36.5 & 17.6 & $<0.001$ \\
\hline & Hospital mortality & 21 & 3.8 & $<0.001$ \\
\hline & $\begin{array}{l}\text { Duration of hospitalization } \\
\text { (median days, [IQR]) }\end{array}$ & $8.6[3.9-18.6]$ & $3.0[1.8-6.5]$ & $<0.001$ \\
\hline \multirow{2}{*}{ Jiajia Qu et al. [9] } & Fever & 78.5 & 89.2 & $<0.05$ \\
\hline & Persistent fever & 50.4 & 74.2 & $<0.01$ \\
\hline \multirow{5}{*}{ Jianguo Zhang et al. [10] } & Cough & 69.7 & 86.1 & 0.001 \\
\hline & Expectoration & 22.7 & 74.8 & $<0.001$ \\
\hline & Dyspnea & 14.7 & 27.8 & 0.004 \\
\hline & Chest pain & 13.7 & 27.8 & 0.002 \\
\hline & Vomiting & 1.4 & 9.6 & $<0.001$ \\
\hline \multirow{17}{*}{ Helene Faury et al. [11] } & Chronic pulmonary diseases & 12.0 & 27.0 & 0.01 \\
\hline & Overweight/Obesity & 40.8 & 25.0 & 0.02 \\
\hline & Median BMI & 27.3 & 24.8 & 0.04 \\
\hline & Fatigue & 63.6 & 39.0 & 0.0006 \\
\hline & Diarrhea & 25.8 & 13.0 & 0.03 \\
\hline & Faintness & 12.1 & 3.0 & 0.02 \\
\hline & Anosmia/Ageusia & 7.0 & 0 & 0.01 \\
\hline & Sputum production & 12.0 & 36.0 & 0.0001 \\
\hline & Nasal Congestion & 8.3 & 21 & 0.02 \\
\hline & Secondary respiratory failure & 21.0 & 0 & $<0.0001$ \\
\hline & Acute Kidney failure & $\mathbf{1 7 . 0}$ & 7.0 & 0.048 \\
\hline & Pulmonary embolism & 6.0 & 0 & 0.03 \\
\hline & Heart congestion & 2.0 & 14.0 & 0.003 \\
\hline & Admitted to ICU & 31.0 & 12.0 & 0.002 \\
\hline & Duration of hospitalization (days, [IQR]) & 10 [4-17] & $4[1-11]$ & $<0.0001$ \\
\hline & Oxygen therapy & 65.0 & 42.3 & 0.002 \\
\hline & Mortality rate & 20.0 & 5.0 & 0.002 \\
\hline \multirow{12}{*}{ Pengfei Li et al. [5] } & Cardiovascular disease/Hypertension & 28.76 & 14.11 & $<0.0001$ \\
\hline & Diabetes & 16.38 & 11.12 & 0.012 \\
\hline & Asthma & 8.42 & 16.09 & 0.0033 \\
\hline & Chronic Obstructive Pulmonary disease & 4.93 & 9.52 & 0.0003 \\
\hline & Immunocompromised conditions & 4.39 & 9.99 & $<0.0001$ \\
\hline & Fever & 72.08 & 89.99 & $<0.0001$ \\
\hline & Cough & 57.99 & 85.31 & $<0.0001$ \\
\hline & Shortness of breath & 32.89 & 49.19 & 0.0249 \\
\hline & Rhinorrhea & 8.48 & 38.57 & $<0.0001$ \\
\hline & Sore throat & 9.48 & 37.28 & $<0.0001$ \\
\hline & Myalgia/Muscle pain & 18.97 & 30.12 & 0.0242 \\
\hline & Vomiting & 8.67 & 24.27 & $<0.0001$ \\
\hline Mengqi Liu et al. [12] & Stuffy and runny nose & 7 & 23 & 0.002 \\
\hline \multirow{4}{*}{ Xiao Tang et al. [13] } & Productive cough & 53.4 & 78.7 & 0.002 \\
\hline & Fatigue & 63 & 18.7 & $<0.001$ \\
\hline & GI symptoms & 37 & 6.7 & $<0.001$ \\
\hline & Myalgia & 34.2 & 14.7 & 0.007 \\
\hline \multirow{2}{*}{ Natalie L. Cobb et al. [14] } & ARDS & 63 & 26 & $<0.001$ \\
\hline & Hospital mortality & 40 & 19 & 0.006 \\
\hline
\end{tabular}


Table 2. Cont.

\begin{tabular}{|c|c|c|c|c|}
\hline References & Significant Clinical Features/Outcome & COVID-19 (\%) & Influenza $(\%)$ & $p$-Value $<0.05$ \\
\hline \multirow{15}{*}{ Souheil Zayet et al. [15] } & Frontal headache & 25.7 & 9.3 & 0.021 \\
\hline & Retro-orbital or temporal headache & 18.6 & 3.7 & 0.013 \\
\hline & Fever & 75.7 & 92.6 & 0.042 \\
\hline & Anosmia & 52.9 & 16.7 & $<0.001$ \\
\hline & Dysgeusia & 48.6 & 20.4 & 0.001 \\
\hline & Diarrhea & 40 & 20.4 & 0.021 \\
\hline & Sputum Production & 28.6 & 51.9 & 0.01 \\
\hline & Sneezing & 18.6 & 46.3 & 0.001 \\
\hline & Dyspnea & 34.3 & 59.3 & 0.007 \\
\hline & Sore throat & 20 & 44.4 & 0.006 \\
\hline & Conjunctival hyperemia & 4.3 & 29.6 & $<0.001$ \\
\hline & Tearing & 5.7 & 24.1 & 0.004 \\
\hline & Vomiting & 2.8 & 22.2 & 0.001 \\
\hline & Crackling sound & 38.6 & 20.4 & 0.032 \\
\hline & Ronchi sounds & 1.4 & 16.7 & 0.002 \\
\hline Hao Wang et al. [16] & Cough & 30.8 & 82.6 & 0 \\
\hline \multirow{6}{*}{ Raija Auvinen et al. [18] } & Pulmonary Diseases & 18 & 45 & 0.03 \\
\hline & Current smoking & 4 & 30 & 0.008 \\
\hline & Headache & 85 & 52 & 0.004 \\
\hline & ARDS & 93 & 58 & 0.003 \\
\hline & ICU admission & 29 & 6 & 0.034 \\
\hline & Duration of hospitalization (days, [IQR]) & $6[4-21]$ & $3[2-4]$ & $<0.001$ \\
\hline \multirow{2}{*}{ Zhilan Yin et al. [19] } & Cough & 73.3 & 96.7 & 0.026 \\
\hline & Expectoration & 43.3 & 80 & 0.007 \\
\hline \multirow{6}{*}{ Yi-Hua Lin et al. [20] } & Fever $38.0^{\circ} \mathrm{C}-38.9^{\circ} \mathrm{C}$ & 43 & 32 & 0.014 \\
\hline & Fever $\geq 39.0^{\circ} \mathrm{C}$ & 11 & 45 & 0.014 \\
\hline & Cough & 51 & 100 & $<0.001$ \\
\hline & Expectoration & 28 & 91 & $<0.001$ \\
\hline & Dyspnea & 9 & 59 & $<0.001$ \\
\hline & Chills & 23 & 55 & 0.015 \\
\hline Jaehee Lee et al. [21] & Median heart rate (bpm) & 83 & 107 & 0.017 \\
\hline
\end{tabular}

Bold: illness (COVID-19 or influenza) with significant difference. Abbreviations: ARDS: Acute respiratory distress syndrome; ICU: Intensive care unit; IQR: interquartile range.

Concerning comorbidities; cardiovascular disease/hypertension, diabetes and obesity were significantly higher in patients with COVID-19, while respiratory diseases such as asthma and chronic obstructive pulmonary disease (COPD) and immunocompromised conditions were significantly more common in influenza patients [5,11,18]. Zayet et al. concluded that COVID-19 patients had a lower Charlson comorbidity index (CCI) than influenza patients $(2 \pm 2.5$ vs. $3 \pm 2.4$, respectively, $p=0.003)$, but with no significant differences regarding comorbidities [15]. Qu et al. reported that while the incidences of COVID-19 and influenza were comparable among the 18-65 and >65 year age groups, the incidences of influenza were much higher than COVID-19 among those aged under 18 years old [9]. However, Lee et al., reported that the median age was significantly higher in patients with COVID-19 compared to patients with influenza (68 (IQR: 59-75) years vs. 57 (IQR: 44-63) years, $p=0.016$ ) [21]. No significant difference was found concerning other demographic characteristics such as gender and ethnicity in our review.

Only a few studies (2/18 studies in adults) were interested in describing the timeline and onset of the main symptoms in COVID-19 and influenza [14,15]. Zayet et al. reconstituted the natural history of the two viruses and suggested that the onset of symptoms (pain symptoms appeared first, followed by fever, cough and diarrhea) did not differ between the two groups except fever, which appeared earlier in COVID-19 than in influenza (1.9 days vs. 2.5 days, $p=0.045$ ) [15]. They also concluded that hospitalization and clinical aggravation occurred later in COVID-19 than in influenza. In fact, COVID-19 patients were hospitalized 
at day $7 \pm 3$ (vs. day $5 \pm 2$ in influenza, $p=0.038$ ), had a respiratory rate $\geq 22 / \mathrm{min}$ at day $9 \pm 0.8$ (vs. day $5 \pm 1.3$ in influenza, $p<0.001$ ), and were admitted to intensive care unit (ICU) at day $10 \pm 2.7$ (vs. day $7 \pm 2.4$ in influenza, $p<0.004$ ). Cobb et al. also reported that patients with COVID-19 had a longer median symptom duration prior to hospitalization compared to patients with influenza (7 days (IQR 5-13) vs. 3.5 (IQR 2-7), $p<0.001$ ) [14]. In the prospective study of Raija Auvinen et al., in multivariable Cox regression analysis, the predictors associated with a longer duration of hospitalization were COVID-19 (hazard ratio (HR) 0.221, $(95 \% \mathrm{CI}=0.118-0.416), p<0.001)$, age ( $\mathrm{HR}=0.972,(0.955-0.990), p=0.002)$, Body Mass Index (HR $=0.950(0.916-0.986), p=0.006)$ and diabetes (HR 0.539, (0.272-1.066), $p=0.076)[18]$.

\subsubsection{Laboratory Findings}

A total of 11 studies of 18 [7-11,13,14,18-21] compared routine laboratory test results between COVID-19 and influenza patients. Both infections caused abnormalities in biological parameters leading to aberrant blood cell counts and sometimes elevated hepatic, renal, and cardiac enzyme activity. White blood cells, especially neutrophils (NE) were significantly more elevated in influenza than COVID-19 patients [7,8,10,11,14,18-21]. Lymphocytes proportion in white blood cell count are higher in influenza than COVID-19 patients $[7,8,20]$ but without difference between lymphocytes level $[7,8]$, except for one study [20]. Platelets level was lower [11] with more often thrombocytopenia [18] in COVID19 than influenza patients. Elevated transaminases were significantly more common in COVID-19 patients $[7,8,11,18,20]$. In a prospective study by Raija Auvinen et al, it was reported that $\mathrm{C}$-reactive protein (CRP) values were similar at admission but rose significantly higher in COVID-19 patients than influenza patients during hospitalization [18]. However, elevated procalcitonin was significantly more frequent in influenza patients than in COVID-19 patients [19,20]. COVID-19 patients had also a greater disposition to elevated prothrombin time (OR $=0.63,95 \% \mathrm{CI}(0.46-0.86), p=0.004)$ [13].

Finally, Chen et al. developed a diagnostic formula to differentiate between COVID19 and influenza during their early stages. Two parameters (monocyte (MO) count and percentage of basophils (BA)) were combined to clarify the diagnostic efficacy, with a sensitivity of $71.6 \%$ and a specificity of $74.8 \%$ (joint probability $(P)=2.388 \times \mathrm{BA} \%-5.182$ $\times$ MO\# + 2.192 (AUC 0.772, 95\% CI (0.718-0.826)). COVID-19 should be considered as the diagnosis when the joint probability is greater than 0.45 , while influenza should be considered when it is less than 0.45 [8] (Table 3).

\subsubsection{Radiological Findings}

We found 10 studies of 18 on adult patients [10-14,16-20] that described imaging findings in COVID-19 and influenza, especially in chest computed tomography (CT) manifestations. Ground-grass opacities (GGO), interlobular septal thickening, and a peripheral distribution were more common in patients with COVID-19 than in patients with influenza $[10,12,13,16,18,20]$. However, consolidation, nodules, and linear opacities were more common in patients with influenza than those with COVID-19 [11-13,18,19]. Tang et al. reported that influenza patients were more inclined to have consolidation manifested on chest $\mathrm{CT}$ imaging $(\mathrm{OR}=4.95(95 \% \mathrm{CI}=1.518-16.176), p=0.008)$, whereas COVID-19 patients had a greater disposition for having GGO on chest CT scans (OR $=0.086(0.015-0.490)$; $p=0.006$ ) [13]. In a review from Onigbinde et al., GGO were usually peripherally located and that vascular engorgement, pleural thickening, and subpleural lines were more frequent in COVID-19 patients. Pneumomediastinum and pneumothorax were only reported in influenza studies [3] (Table 4). 
Table 3. Significant laboratory findings in COVID-19 and influenza adult groups.

\begin{tabular}{|c|c|c|c|c|}
\hline References & Significant Laboratory Findings & COVID-19 (\%) & Influenza (\%) & $p$-Value $<0.05$ \\
\hline \multirow{45}{*}{ Ying Luo et al. [7] } & $\begin{array}{l}\text { White blood cell count }\left(\times 10^{9} / \mathrm{L} \text {, }\right. \\
\text { median, [IQR] })\end{array}$ & 5.45 [4.46-7.17] & $6.14[4.66-8.24]$ & $<0.001$ \\
\hline & Neutrophil $\left(\times 10^{9} / \mathrm{L}\right.$, median, $\left.\left.[\mathrm{IQR}]\right)\right)$ & $3.68[2.68-5.16]$ & $4.09[2.85-6.11]$ & $<0.001$ \\
\hline & Lymphocyte $(\%$, median, [IQR] $)$ & $22.0[14.6-29.4]$ & $20.5[13.3-28.6]$ & 0.009 \\
\hline & Monocyte $\left(\times 10^{9} / \mathrm{L}\right.$, median, [IQR]) & $0.47[0.34-0.61]$ & $0.52[0.37-0.69]$ & $<0.001$ \\
\hline & Eosinophil $\left(\times 10^{9} / \mathrm{L}\right.$, median, [IQR] $)$ & $0.01[0.00-0.05]$ & $0.02[0.00-0.07]$ & $<0.001$ \\
\hline & Eosinophil (\%, median, [IQR]) & $0.2[0.0-2.9]$ & $0.3[0.0-1.2]$ & $<0.001$ \\
\hline & Basophil (\%, median, [IQR]) & $0.2[0.0-0.3]$ & $0.2[0.1-0.3]$ & $<0.001$ \\
\hline & $\begin{array}{l}\text { Red blood cell count }\left(\times 10^{12} / \mathrm{L},\right. \\
\text { median, }[\mathrm{IQR}])\end{array}$ & $4.43[4.00-4.84]$ & 4.37 [3.96-4.78] & 0.012 \\
\hline & Hemoglobin (g/L, median, [IQR]) & 134 [122-146] & 131 [119-143] & $<0.001$ \\
\hline & Hematocrit $(\%$, median, [IQR]) & $39.7[36.2-43.1]$ & $39.1[35.5-42.4]$ & 0.002 \\
\hline & MCV (fL, median, [IQR]) & 89.1 [86.4-91.7] & $89.6[86.7-92.4]$ & 0.003 \\
\hline & $\mathrm{MCH}(\mathrm{pg}$, median, [IQR]) & $30.6[29.5-31.6]$ & $30.4[29.3-31.3]$ & 0.002 \\
\hline & MCHC (g/L, median, [IQR]) & $343[335-351]$ & 337 [329-346] & $<0.001$ \\
\hline & RDW-CV (Median, [IQR]) & $12.2[11.9-12.8]$ & $12.5[12.0-13.2]$ & $<0.001$ \\
\hline & RDW-SD (fL, median, [IQR]) & $39.5[37.8-41.8]$ & $40.9[38.8-43.2]$ & $<0.001$ \\
\hline & PDW (fL, median, [IQR]) & $12.0[10.8-13.6]$ & $12.3[11.0-13.9]$ & 0.021 \\
\hline & $\begin{array}{l}\text { Alanine aminotransferase }(\mathrm{U} / \mathrm{L}, \\
\text { median, }[\mathrm{IQR}])\end{array}$ & $25[18-38]$ & $24[16-36]$ & 0.019 \\
\hline & $\begin{array}{c}\text { Aspartate aminotransferase }(\mathrm{U} / \mathrm{L}, \\
\text { median, }[\mathrm{IQR}])\end{array}$ & $27[21-36]$ & 25 [19-35] & $<0.001$ \\
\hline & Total Protein $(\mathrm{g} / \mathrm{L}$, mean $)$ & $69.3 \pm 5.6$ & $68.5 \pm 6.4$ & 0.003 \\
\hline & Globulin (g/L, median, [IQR]) & $32.4 \pm 4.4$ & $31.8 \pm 4.8$ & $<0.001$ \\
\hline & $\begin{array}{c}\text { Indirect Bilirubin }(\mu \mathrm{mol} / \mathrm{L}, \\
\text { median, }[\mathrm{IQR}])\end{array}$ & $5.5[4.2-7.3]$ & $4.9[3.8-6.9]$ & $<0.001$ \\
\hline & GGT (U/L, median, [IQR]) & $30[21-48]$ & $35[21-54]$ & 0.003 \\
\hline & $\begin{array}{c}\text { Alkaline Phosphatase }(\mathrm{U} / \mathrm{L}, \\
\text { median, [IQR]) }\end{array}$ & $65[56-78]$ & $75[63-96]$ & $<0.001$ \\
\hline & LDH (U/L, median, [IQR]) & 260 [217-327] & 235 [196-298] & $<0.001$ \\
\hline & Triglyceride (mmol/L, median, $[\mathrm{IQR}])$ & $1.75 \pm 088$ & $1.63 \pm 0.84$ & $<0.001$ \\
\hline & HDL-C (mmol/L, median, [IQR]) & $0.99 \pm 0.19$ & $0.97 \pm 0.22$ & 0.002 \\
\hline & LDL-C (mmol/L, median, [IQR]) & $2.45 \pm 0.55$ & $2.41 \pm 0.68$ & 0.004 \\
\hline & Creatinine $(\mu \mathrm{mol} / \mathrm{L}$, median, $[\mathrm{IQR}])$ & $72[61-87]$ & 69 [59-82] & $<0.001$ \\
\hline & Urea $(\mathrm{mmol} / \mathrm{L}$, median, $[\mathrm{IQR}])$ & $5.89 \pm 3.84$ & $5.54 \pm 3.41$ & 0.001 \\
\hline & Uric acid $(\mu \mathrm{mol} / \mathrm{L}$, median, $[\mathrm{IQR}])$ & $253[206-313]$ & $260[219-304]$ & 0.031 \\
\hline & Calcium (mmol/L, median, [IQR]) & $2.14 \pm 0.11$ & $2.17 \pm 0.11$ & $<0.001$ \\
\hline & Magnesium (mmol/L, median, [IQR]) & $0.87 \pm 0.07$ & $0.86 \pm 0.09$ & 0.001 \\
\hline & Chlorine (mmol/L, median, [IQR]) & $100.4 \pm 4.2$ & $101.4 \pm 3.7$ & $<0.001$ \\
\hline & Potassium (mmol/L, median, [IQR]) & $4.21 \pm 0.42$ & $4.15 \pm 0.40$ & $<0.001$ \\
\hline & Sodium (mmol/L, median, [IQR]) & $139.7 \pm 3.9$ & $139.1 \pm 3.4$ & $<0.001$ \\
\hline & Phosphate (mmol/L, median, [IQR]) & $1.04 \pm 0.26$ & $1.05 \pm 0.20$ & 0.002 \\
\hline & $\mathrm{HCO}^{3}(\mathrm{mmol} / \mathrm{L}$, median, [IQR]) & $24.5 \pm 2.9$ & $24.0 \pm 3.1$ & $<0.001$ \\
\hline & $\begin{array}{l}\text { Hypersensitive CRP }(\mathrm{mg} / \mathrm{L}, \\
\text { median, [IQR]) }\end{array}$ & $20.0[5.8-45.8]$ & $15.7[4.8-40.1]$ & 0.024 \\
\hline & $\operatorname{ESR}(\mathrm{mm} / \mathrm{h}$, median, $[\mathrm{IQR}])$ & $35[24-47]$ & $27[17-40]$ & $<0.001$ \\
\hline & Prothrombin time (s, mean) & $14.06 \pm 1.09$ & $14.09 \pm 1.83$ & $<0.001$ \\
\hline & $\operatorname{APTT}(\mathrm{s}$, mean $)$ & $39.9 \pm 4.5$ & $39.6 \pm 5.0$ & 0.02 \\
\hline & Thrombin time (s, mean) & $16.9 \pm 1.4$ & $16.6 \pm 2.0$ & $<0.001$ \\
\hline & Prothrombin activity $(\%$, mean $)$ & $91 \pm 11$ & $92 \pm 14$ & $<0.001$ \\
\hline & Fibrinogen (g/L, mean) & $4.71 \pm 1.08$ & $4.27 \pm 1.18$ & $<0.001$ \\
\hline & D-Dimer (mg/L, median, [IQR]) & $1.24[0.65-2.75]$ & $1.72[0.85-3.30]$ & $<0.001$ \\
\hline
\end{tabular}


Table 3. Cont.

\begin{tabular}{|c|c|c|c|c|}
\hline References & Significant Laboratory Findings & COVID-19 (\%) & Influenza (\%) & $p$-Value $<0.05$ \\
\hline \multirow{7}{*}{ Jiangnan Chen et al. [8] } & Monocyte $\left(\times 10^{9} / \mathrm{L}\right.$, median, [IQR]) & $0.36[0.28-0.48]$ & $0.55[0.4-0.71]$ & 0 \\
\hline & Monocyte $(\%$, median, $[\mathrm{IQR}])$ & $7.60[6.20-9.95]$ & $9.0[7.20-11.40]$ & 0 \\
\hline & Neutrophil $\left(\times 10^{9} / \mathrm{L}\right.$, median, [IQR]) & $2.93[2.26-3.79]$ & $4.26[3.00-5.74]$ & 0 \\
\hline & Neutrophil $(\%$, mean $)$ & $64.50 \pm 11.64$ & $68.42 \pm 14.69$ & 0.011 \\
\hline & Lymphocyte $(\%$, mean $)$ & $26.30 \pm 10.52$ & $21.07 \pm 12.85$ & 0 \\
\hline & Eosinophil (\%, median, [IQR]) & $0.60[0.30-1.15]$ & $0.40[0.10-1.10]$ & 0.038 \\
\hline & Basophil (\%, median, [IQR]) & $0.20[0.10-0.30]$ & $0.10[0.10-0.30]$ & 0.001 \\
\hline \multirow{5}{*}{ Jiajia Qu et al. [9] } & Elevated lymphocyte & 0 & 5 & $<0.01$ \\
\hline & Abnormal Urinary test & 32.11 & 21.67 & $<0.05$ \\
\hline & Urine protein positive & 16.26 & 8.33 & $<0.05$ \\
\hline & Elevated procalcitonin & 40.83 & 10.98 & $<0.01$ \\
\hline & Elevated white blood cells & 75 & 26.83 & $<0.01$ \\
\hline \multirow{4}{*}{ Jianguo Zhang et al. [10] } & Leukocytosis > $9.5 \times 10^{9} / \mathrm{L}$ & 16.1 & 30.4 & 0.003 \\
\hline & Neutrophilia $>75 \%$ & 32.2 & 50.4 & 0.001 \\
\hline & Lymphocytopenia $<20 \%$ & 46.9 & 68.7 & $<0.001$ \\
\hline & Creatine Kinase $>25 \mathrm{U} / \mathrm{L}$ & 11.8 & 3.5 & 0.013 \\
\hline \multirow{8}{*}{ Helene Faury et al. [11] } & $\begin{array}{l}\text { White Blood cell count (G/L, median, } \\
[\mathrm{IQR}])\end{array}$ & $5.88[4.41-7.68]$ & $6.72[5.15-9.42]$ & 0.01 \\
\hline & Neutrophil (G/L, median, [IQR]) & $4.11[2.99-5.65]$ & $5.06[3.43-7.25]$ & 0.02 \\
\hline & Platelets $(\mathrm{G} / \mathrm{L}$, median, [IQR]) & $179[145-225]$ & 199 [168-239] & 0.04 \\
\hline & Sodium (U/L, median, [IQR])) & $137[135-139]$ & $138[136-140]$ & 0.006 \\
\hline & Troponin (ng/L, median, [IQR]) & $9.2[6.5-22.4]$ & $34.4[8.8-72.2]$ & 0.007 \\
\hline & Albumin (g/L, median, [IQR]) & $30[27-33]$ & $37[33-39]$ & 0.04 \\
\hline & $\begin{array}{c}\text { Aspartate aminotransferase }(\mathrm{U} / \mathrm{L}, \\
\text { median, }[\mathrm{IQR}])\end{array}$ & $45[34-76]$ & 34 [29-49] & 0.02 \\
\hline & LDH $(\mathrm{U} / \mathrm{L}$, median, $[\mathrm{IQR}])$ & $397[305-544]$ & $298[248-383]$ & 0.04 \\
\hline \multirow{6}{*}{ Xiao Tang et al. [13] } & $\mathrm{PaO}_{2} / \mathrm{FiO}_{2}$ (Median, $\mathrm{mm} \mathrm{Hg}$ ) & 198.5 & 107 & $<0.001$ \\
\hline & Aspartate transaminase $(\mathrm{U} / \mathrm{L})$ & 25.5 & 70 & $<0.001$ \\
\hline & LDH (U/L) & 483 & 767 & $<0.001$ \\
\hline & Troponin I (ng/mL) & 0.03 & 0.14 & $<0.001$ \\
\hline & $\mathrm{CD}^{+}$(Median, cells $/ \mathrm{mL}$ ) & 193 & 303 & 0.007 \\
\hline & $\mathrm{CD}^{+} / \mathrm{CD}^{+}$(Median, cells $\left./ \mathrm{mL}\right)$ & 97 & 185 & $<0.001$ \\
\hline \multirow{3}{*}{ Natalie L. Cobb et al. [14] } & $\begin{array}{l}\text { White blood cells at admission } \\
\text { (median, [IQR]) }\end{array}$ & $\begin{array}{c}7240 \\
{[5430-11,820]}\end{array}$ & $\begin{array}{c}9035 \\
{[6590-14,900]}\end{array}$ & 0.007 \\
\hline & $\begin{array}{l}\text { Neutrophil at admission } \\
\text { (median, [IQR]) }\end{array}$ & $5405[3880-9580]$ & $\begin{array}{c}7210 \\
{[4990-11,890]}\end{array}$ & 0.02 \\
\hline & Early sputum cultures (positive) & 27.2 & 72.2 & 0.005 \\
\hline \multirow{4}{*}{ Raija Auvinen et al. [18] } & $\begin{array}{l}\text { Leukocytes count } \times 10^{9} / \mathrm{L} \\
(\text { Median, }[\mathrm{IQR}])\end{array}$ & $5.1(4.0-6.3)$ & $6.7(5.4-10.9)$ & 0.002 \\
\hline & Leukocytosis & 11 & 39 & 0.019 \\
\hline & Thrombocytopenia $<150 \times 10^{9} / \mathrm{L}$ & 39 & 12 & 0.019 \\
\hline & Alanine aminotransferase (U/L, [IQR]) & $42[19-127]$ & $23[12-123]$ & 0.011 \\
\hline \multirow[t]{2}{*}{ Zhilan Yin et al. [19] } & $\begin{array}{l}\text { Neutrophil }\left(\times 10^{9} \text { cells } / \mathrm{L}\right. \\
\text { median, }[\mathrm{IQR}])\end{array}$ & $3.57[2.72-4.92]$ & $4.75[3.15-7.00]$ & 0.037 \\
\hline & Procalcitonin (ng/mL, median, [IQR]) & $0.04[0.03-0.09]$ & $0.11[0.09-0.37]$ & 0.002 \\
\hline
\end{tabular}


Table 3. Cont

\begin{tabular}{|c|c|c|c|c|}
\hline References & Significant Laboratory Findings & COVID-19 (\%) & Influenza (\%) & $p$-Value $<0.05$ \\
\hline \multirow{10}{*}{ Yi-Hua Lin et al. [20] } & White blood cells $\left(\times 10^{9}\right.$ cells $/ \mathrm{L}$, mean $)$ & $4.87 \pm 2.04$ & $7.59 \pm 5.12$ & 0.026 \\
\hline & Leukocytosis & 3 & 32 & 0.002 \\
\hline & Neutrophil (×109 /L, mean) & $3.16 \pm 1.73$ & $6.20 \pm 4.84$ & 0.009 \\
\hline & Lymphocyte $\left(\times 10^{9} / \mathrm{L}\right.$, mean $)$ & $1.19 \pm 0.59$ & $0.88 \pm 0.52$ & 0.049 \\
\hline & Anemia & 0 & 41 & $<0.001$ \\
\hline & CRP (mg/L, median, [IQR])) & $9.56[3.82-22.42]$ & $\begin{array}{c}55.3 \\
{[33.97-102.77]}\end{array}$ & 0.001 \\
\hline & Procalcitonin (ng/L, median, [IQR]) & $0.05[0.05-0.06]$ & $0.25[0.08-2.28]$ & $<0.001$ \\
\hline & Urea Nitrogen (mmol/L, mean) & $3.76 \pm 1.37$ & $6.36 \pm 3.30$ & 0.002 \\
\hline & LDH (U/L, median, [IQR])) & $\begin{array}{c}158.0 \\
{[142.0-196.0]}\end{array}$ & $\begin{array}{c}243.5 \\
{[198.3-328.8]}\end{array}$ & $<0.001$ \\
\hline & $\mathrm{PaO}_{2} / \mathrm{FiO}_{2}<200 \mathrm{~mm} \mathrm{Hg}$ & 4 & 22 & 0.022 \\
\hline Jaehee Lee et al. [21] & White blood cell (Median, cells/uL) & 7470 & 2680 & 0.027 \\
\hline
\end{tabular}

Bold: illness (COVID-19 or influenza) with significant difference. Abbreviations: Leukocytosis: leukocyte count $>10 \times 10^{9} / \mathrm{L}$; CRP: C-reactive protein; LDH: Lactate dehydrogenase; IQR: Interquartile range; MCV: Mean corpuscular volume; MCH: Mean corpuscular hemoglobin; MCHC: Mean corpuscular concentration; RDW-CV: Coefficient variation of red blood cell volume width; RDW_SD: Standard deviation in red cell distribution width; PDW: Platelet distribution width; GGT: $\gamma$-glutamyl transpeptidase; HDL-C: High-density lipoprotein cholesterol; LDL-C: Low-density lipoprotein cholesterol; $\mathrm{HCO}_{3}$ : Bicarbonate ion; ESR: Erythrocyte sedimentation rate; APTT: Activated partial thromboplastic time.

Table 4. Significant radiological findings between COVID-19 and influenza adult groups.

\begin{tabular}{|c|c|c|c|c|}
\hline References & Significant Radiological Findings & COVID-19 (\%) & Influenza (\%) & $p$-Value $<0.05$ \\
\hline \multirow{8}{*}{ Jianguo Zhang et al. [10] } & Rounded opacities & 37.9 & 19.1 & $<0.001$ \\
\hline & Bronchiolar wall thickening & 33.6 & 13 & $<0.0001$ \\
\hline & Air bronchogram & 29.9 & 13 & $<0.001$ \\
\hline & Consolidation & 26.1 & 15.7 & 0.031 \\
\hline & Interlobular septal thickening & 24.2 & 13.9 & 0.029 \\
\hline & Crazy paving pattern & 22.3 & 9.6 & 0.004 \\
\hline & Tree-in-bud & 13.7 & 5.2 & 0.018 \\
\hline & GGO with consolidation & 25.6 & 39.1 & 0.011 \\
\hline Helene Faury et al. [11] & Pulmonary nodules & 8.8 & 50.0 & 0.001 \\
\hline \multirow{14}{*}{ Mengqi Liu et al. [12] } & Predominant distribution: & & & \\
\hline & - Central & 2 & 6 & 0.022 \\
\hline & - Peripheral & 45 & 20 & \\
\hline & - Mixed & 53 & 74 & \\
\hline & Interlobular septal thickening & 66 & 43 & 0.014 \\
\hline & Rounded opacities & 35 & 17 & 0.048 \\
\hline & Nodules & 28 & 71 & $<0.001$ \\
\hline & Tree-in-bud & 9 & 40 & $<0.001$ \\
\hline & Pleural effusion & 6 & 31 & $<0.001$ \\
\hline & Pure GGO without nodules & 29 & 11 & $<0.001$ \\
\hline & Pure GGO + interlobular septal thickening & 21 & 6 & 0.042 \\
\hline & Rounded opacities without nodules & 22 & 0 & 0.002 \\
\hline & $\begin{array}{c}\text { Interlobular septal thickening } \\
\text { without nodules }\end{array}$ & 45 & 6 & $<0.001$ \\
\hline & $\begin{array}{l}\text { Rounded opacities }+ \text { interlobular septal } \\
\text { thickening }+ \text { absence of pleural effusion }\end{array}$ & 19 & 3 & 0.021 \\
\hline \multirow{2}{*}{ Xiao Tang et al. [13] } & GGO & 94.5 & 45.3 & $<0.001$ \\
\hline & Consolidation & 28.8 & 45.3 & 0.042 \\
\hline Natalie L Cobb et al. [14] & Bilateral opacities & 90 & 52 & $<0.001$ \\
\hline
\end{tabular}


Table 4. Cont.

\begin{tabular}{|c|c|c|c|c|}
\hline References & Significant Radiological Findings & COVID-19 (\%) & Influenza $(\%)$ & $p$-Value $<0.05$ \\
\hline \multirow{22}{*}{ Hao Wang et al. [16] } & Lesion Distribution: & & & \\
\hline & - Central & 7.7 & 75 & 0.000 \\
\hline & - Peripheral & 38.5 & 3.3 & \\
\hline & - Diffuse & 0 & 21.7 & \\
\hline & - Non-specific & 53.8 & 0 & \\
\hline & Lobe predomination: & & & \\
\hline & - Superior lobe & 23.1 & 23.9 & 0.001 \\
\hline & - Inferior lobe & 15.4 & 57.6 & \\
\hline & - Middle lobe & 7.7 & 7.6 & \\
\hline & - Balanced predomination & 53.8 & 10.9 & \\
\hline & Lesion margin: & & & \\
\hline & - Clear & 46.2 & 10.9 & 0.004 \\
\hline & - Vague & 53.8 & 89.1 & \\
\hline & GGO Involvement pattern: & & & \\
\hline & - Patchy & 38.5 & 5.4 & 0.000 \\
\hline & - Cluster like & 7.7 & 77.2 & \\
\hline & - GGO + consolidation opacities & 46.2 & 6.5 & \\
\hline & - Whole consolidation & 7.7 & 10.9 & \\
\hline & Lesion Contour: & & & \\
\hline & -Shrinking & 69.2 & 1.1 & 0.000 \\
\hline & - Non-shrinking & 30.8 & 98.9 & \\
\hline & Bronchial wall thickening & 0 & 32.6 & 0.018 \\
\hline \multirow{8}{*}{ Liaoyi Lin et al. [17] } & Close to the pleura & 69 & 40 & 0.005 \\
\hline & Mucoid impaction & 2 & 13 & 0.047 \\
\hline & Pleural effusion & 0 & 22 & $<0.001$ \\
\hline & Axial distribution: & & & \\
\hline & - Inner & 6 & 7 & $<0.001$ \\
\hline & - Outer & 67 & 24 & \\
\hline & - Diffuse & 12 & 36 & \\
\hline & - Random & 15 & 33 & \\
\hline \multirow{2}{*}{ Raija Auvinen et al. [18] } & Linear opacities & 14 & 42 & 0.024 \\
\hline & GGO/Consolidation & 68 & 21 & $<0.001$ \\
\hline \multirow{6}{*}{ Zhilan Yin et al. [19] } & Vascular enlargement & 67 & 93 & 0.037 \\
\hline & Pleural Thickening & 63 & 90 & 0.03 \\
\hline & Linear opacification & 50 & 90 & 0.002 \\
\hline & Crazy-paving sign & 30 & 60 & 0.021 \\
\hline & Pleural effusion & 53 & 13 & 0.002 \\
\hline & Bronchiectasis & 30 & 3 & 0.012 \\
\hline \multirow{4}{*}{ Yi-Hua Lin et al. [20] } & GGO & 71 & 23 & $<0.001$ \\
\hline & Infiltration & 29 & 68 & 0.003 \\
\hline & GGO + reticular pattern & 63 & 0 & $<0.001$ \\
\hline & Interlobular septal thickening & 71 & 27 & 0.001 \\
\hline
\end{tabular}

Bold: illness (COVID-19 or influenza) with significant difference. Abbreviations: GGO: Ground-glass opacity.

\section{Discussion}

The current review compared the clinical features and courses, laboratory data, and radiological findings between COVID-19 patients and those with influenza. We initially found that compared to patients with influenza, COVID-19 patients were more likely to exhibit symptoms such as diarrhea and neurologic symptoms. However, fever, productive cough, vomiting, dyspnea, ocular and otorhinolaryngological symptoms were more observed in the group influenza than the group COVID-19. Some authors concluded by describing the timeline of these viruses that SARS-CoV-2 infection may present with a slow onset compared with the clinical presentations of influenza infection with a longer incubation period $[13,15]$. Indeed, the differences in the clinical presentation of these two viral infections can be explained, in large part, by the pathophysiological distribution of the entry 
receptors for each virus. Human influenza A virus binds to cell receptors alpha2,6-linked via sialic acid linked glycoproteins. The distribution of sialic acid on cell surfaces is one determinant of host tropism and understanding its expression on human cells and tissues is important for understanding influenza pathogenesis. These receptors were especially expressed on the respiratory tract, from the nasopharynx, trachea to the bronchi, except the alveoli ( $\alpha 2,3$-linked sialic acid receptors predominant on alveolar cells) [22]. The short incubation period of influenza infection with the predominant respiratory manifestations (sore throat, sneezing, sputum production, rhonchi on pulmonary auscultation) is well explained by this distribution. On the other hand, Angiotensin-converting enzyme 2 (ACE2) protein, known as the key regulator enzyme of the renin-angiotensin-aldosterone system (RAAS) is the functional receptor of SARS-CoV-2 and its expression and activity will mediate directly the SARS-CoV-2 infection. Regarding the tissue distribution of ACE2 protein, ACE2 is highly expressed on lung alveolar epithelial cells, small intestinal epithelial cells and endothelial cells (including in the central nervous system), but poorly found on the surface of nasopharyngeal cells [23]. When the SARS spike protein binds to the ACE-2 receptor, the complex is proteolytically processed by type 2 transmembrane protease TMPRSS2 leading to cleavage of ACE-2 and activation of the spike [24]. This mechanism is also described in influenza physiopathology. All these findings trigger a longer incubation period of SARS-CoV-2 infection and the observed symptoms in COVID19 patients of dyspnea, dry cough, diarrhea, and bilateral crackling sound on pulmonary auscultation, but also neurologic symptoms such as new loss of smell and taste. ACE is also abundantly present in the basal layer of the non-keratinizing squamous epithelium of nasal and oral mucosa [25]. Indeed, most reports have so far linked new loss of taste or smell to neurological symptoms instead of rhinolaryngological symptoms [26]. In any case, in this epidemic context, patients presenting with dysgeusia and/or anosmia may be considered as patients infected with COVID-19, until microbiological confirmation has been obtained. In another study including 217 patients presenting influenza-like illness, we demonstrated that the specificity of the combination of anosmia and dysgeusia reached 91\% for a positive SARS-CoV-2 RT-PCR result [27]. Unspecific symptoms such as fever and musculoskeletal symptoms or pain syndrome defined by fatigue, myalgia and/or arthralgia are associated with a cascade of inflammatory mediators and were not directly linked to the distribution of viral receptors. These clinical presentations can be equally described in the two illnesses. Concerning the outcome, both COVID-19 and influenza may be accompanied by ARDS with a high mortality [13]. However, it was clear that mortality and lethality of SARS-CoV-2 infection were much higher than those of the influenza infection in the general population [28].

Comparison of peripheral blood parameters from COVID-19 and influenza patients revealed some differences, especially during the early stages of the disease. Concerning the blood count, NE were significantly more elevated in influenza than COVID-19 patients. Jiangnan Chen et al. conducted their study with a group control [8], the difference about NE is explained by an increase of NE in influenza and a decrease of NE in SARS-CoV-2 infection. The only study which has shown a higher level of lymphocytes in influenza than SARS-CoV-2 infection [20] is not conclusive because the two populations were not comparable ( $18 \%$ received invasive mechanical ventilation (IMV) in the influenza group while none received IMV in the COVID-19 group). Influenza and SARS-CoV-2 infection seems to decrease lymphocytes level with the same intensity. In another short review of COVID-19 hematological manifestations, Slomka et al. showed that the majority of patients (especially those presenting with an ARDS related to SARS-CoV-2) are likely to develop lymphocytopenia. The decreased number of lymphocytes is described in other diseases caused by other coronaviruses primarily infecting the human respiratory tract [29]. This lymphopenia is probably explained by direct infection of lymphocytes and destruction of lymphoid organs caused by the "cytokine storm" and a major release of pro-inflammatory cytokines [30]. On the one hand, it is known that $\mathrm{MO}$ and macrophages play central roles in the immune response of humans and in protecting the body from 
influenza infection. They are necessary for the influenza virus to infect lymphocytes and regulate lymphocyte apoptosis by synthesizing and expressing viral neuraminidase [31]. On the other hand, several studies revealed that COVID-19 patients exhibited a significant decrease under lower limit of lymphocyte counts [32]. This indicates that SARS-CoV2 consumes many immune cells and inhibits the body's cellular immune function [33]. Conversely, inflammatory cytokines including serum amyloid A (SAA), CRP, IL-6, IL-10 were significantly higher than normal values [32]. SARS-COV-2 invasion activated T cellmediated immunity, which resulted in increasing production of inflammatory cytokine [34]. However, inhibition cytokines such as IL-10 can suppress T cell activation conversely. Serum amyloid A and CRP are both of acute-phase proteins in response to inflammatory cytokines related by activated $\mathrm{MO} /$ macrophages after infections. High levels of SAA and CRP could reflect the severity of inflammation and result in a "cytokine storm", during which the inflammation spreads throughout the body via the circulation. This severe inflammatory state secondary to COVID-19 leads to a severe derangement of hemostasis that has been recently described as a state of hypercoagulopathy, defined as increased degradation products such as D-dimer and fibrinogen. In a large American cohort study of 9407 adult COVID-19 patients, the overall in-hospital venous thromboembolism (VTE) was approximately $3 \%$. The authors concluded that key predictors of VTE or mortality included advanced age, increasing CCI, past history of cardiovascular disease, ICU level of care, and elevated level of D-dimer [35]. In addition, several radiologists tried to differentiate COVID19 from Influenza and other viral pneumonia on chest CT images [36]. Usually, the common findings on CT for typical influenza pneumonia consist of diffuse or multifocal GGO and small centrilobular nodules [3]. Onigbinde et al. showed that the occurrence of GGO and consolidation in COVID-19 were not markedly different from that of influenza studies. However, they were predominantly located bilaterally within the lower lobes for COVID-19 patients, whereas in the influenza studies, they were more widespread, involving all the lobes [3]. In another study including 122 patients, Mengqi et al. concluded that the COVID19 patients were more likely to have rounded opacities and interlobular septal thickening compared with the influenza group, but less likely to have nodules, tree-in-bud sign and pleural effusion with significant difference between the two groups [12]. In our review, complications such as pneumothorax and pneumomediastinum were only described in influenza patients. These respiratory complications remain scarce during the course of COVID-19 and have recently been described in medical literature [3,37,38]. Therefore, these differential pathologic changes may present themselves as distinguishing imaging characteristics during clinical assessments. There are some limitations to our review. First of all, COVID-19 is relatively novel with a limited number of patients and studies. In most articles, the clinical features, laboratory data and CT findings reporting on influenza dated before the onset of COVID 19. Finally, the use of abstracts in selecting full texts for a detailed review could have led to the omission of some articles. Moreover, we only considered PubMed a reference for our bibliographic research and database with accessible free access articles. We also just summarized what we found in recent medical literature and focused on what was statistically significant. Finally, a meta-analysis is recommended to further define the differences and the degree between COVID-19 and influenza.

\section{Conclusions}

This study provided a comprehensive comparison of adult patients in SARS-CoV-2 and influenza infections, regarding comorbidities, clinical and paraclinical features, and outcome. Clinical manifestations of COVID-19 and influenza seem to be similar with some differences: neurologic symptoms and diarrhea were more described in COVID-19, however; vomiting, ocular and otorhinolaryngological symptoms were more observed in influenza infection. Both viruses decreased lymphocytes; NE were significantly more elevated in influenza than COVID-19 patients while elevated transaminases were significantly more elevated in COVID-19 than influenza patients. Radiological findings showed that GGO are usually peripherally located in COVID-19 compared with influenza which 
also had central and random locations. All these findings can help clinicians when dealing with cases of influenza-like illnesses during the period of co-circulation of influenza and SARS-CoV-2.

Author Contributions: Conceptualization, M.O., T.K. and S.Z.; methodology, M.O.; software, M.O. and S.Z.; validation, M.O., T.K., N.B., V.G. and S.Z.; formal analysis, M.O.; investigation, M.O. and S.Z.; resources, M.O.; data curation, M.O.; writing—original draft preparation, M.O. and S.Z.; writing-review and editing, M.O., T.K. and S.Z.; visualization, N.B. and V.G.; supervision, T.K., N.B., V.G. and S.Z.; project administration, M.O.; funding acquisition, M.O. All authors have read and agreed to the published version of the manuscript.

Funding: This research received no external funding.

Institutional Review Board Statement: Due to the nature of the study (review), the Ethics \& Scientific Committee of Nord Franche-Comté Hospital determined that patient consent was required for publication. We make sure to keep patient data confidential and in compliance with the Declaration of Helsinki.

\section{Informed Consent Statement: Not applicable.}

Data Availability Statement: Data available on request due privacy to restrictions. The data presented in this case study are available on request from the corresponding author.

Conflicts of Interest: The authors declare no conflict of interest. The funders had no role in the design of the study; in the collection, analyses, or interpretation of data; in the writing of the manuscript, or in the decision to publish the results.

\section{References}

1. Zhu, N.; Zhang, D.; Wang, W.; Li, X.; Yang, B.; Song, J.; Zhao, X.; Huang, B.; Shi, W.; Lu, R.; et al. A Novel Coronavirus from Patients with Pneumonia in China, 2019. N. Engl. J. Med. 2020, 382, 727-733. [CrossRef]

2. Khan, M.S.; Shahid, I.; Anker, S.D.; Solomon, S.D.; Vardeny, O.; Michos, E.D.; Fonarow, G.C.; Butler, J. Cardiovascular implications of COVID-19 versus influenza infection: A review. BMC Med. 2020, 18, 403. [CrossRef]

3. Onigbinde, S.O.; Ojo, A.S.; Fleary, L.; Hage, R. Chest Computed Tomography Findings in COVID-19 and Influenza: A Narrative Review. Biomed Res. Int. 2020, 2020, 6928368. [CrossRef]

4. WHO. Up to 650000 People Die of Respiratory Diseases Linked to Seasonal Flu Each Year. Available online: https://www.who. int/news/item/13-12-2017-up-to-650-000-people-die-of-respiratory-diseases-linked-to-seasonal-flu-each-year (accessed on 15 January 2021).

5. Li, P.; Wang, Y.; Peppelenbosch, M.P.; Ma, Z.; Pan, Q. Systematically comparing COVID-19 with 2009 influenza pandemic for hospitalized patients. Int. J. Infect. Dis. 2020, 102, 375-380. [CrossRef]

6. Cates, J.; Lucero-Obusan, C.; Dahl, R.M.; Schirmer, P.; Garg, S.; Oda, G.; Hall, A.J.; Langley, G.; Havers, F.P.; Holodniy, M.; et al. Risk for In-Hospital Complications Associated with COVID-19 and Influenza-Veterans Health Administration, United States, October 1, 2018-May 31, 2020. Morb. Mortal. Wkly. Rep. 2020, 69, 1528-1534. [CrossRef]

7. Luo, Y.; Yuan, X.; Xue, Y.; Mao, L.; Lin, Q.; Tang, G.; Song, H.; Liu, W.; Hou, H.; Wang, F.; et al. Using a diagnostic model based on routine laboratory tests to distinguish patients infected with SARS-CoV-2 from those infected with influenza virus. Int. J. Infect. Dis. 2020, 95, 436-440. [CrossRef]

8. Chen, J.; Pan, Y.; Li, G.; Xu, W.; Zhang, L.; Yuan, S.; Xia, Y.; Lu, P.; Zhang, J. Distinguishing between COVID-19 and influenza during the early stages by measurement of peripheral blood parameters. J. Med. Virol. 2020. [CrossRef] [PubMed]

9. Qu, J.; Chang, L.K.; Tang, X.; Du, Y.; Yang, X.; Liu, X.; Han, P.; Xue, Y. Clinical characteristics of COVID-19 and its comparison with influenza pneumonia. Acta Clin. Belg. Int. J. Clin. Lab. Med. 2020, 75, 348-356. [CrossRef] [PubMed]

10. Zhang, J.J.; Ding, D.; Huang, X.; Zhang, J.J.; Chen, D.; Fu, P.; Shi, Y.; Xu, W.; Tao, Z. Differentiation of COVID-19 from seasonal influenza: A multicenter comparative study. J. Med. Virol. 2020. [CrossRef] [PubMed]

11. Faury, H.; Courboulès, C.; Payen, M.; Jary, A.; Hausfater, P.; Luyt, C.E.; Dres, M.; Pourcher, V.; Abdi, B.; Wirden, M.; et al. Medical Features of COVID-19 and Influenza Infection: A Comparative Study in Paris, France. J. Infect. 2020, 82, E36-E39. [CrossRef] [PubMed]

12. Liu, M.; Zeng, W.; Wen, Y.; Zheng, Y.; Lv, F.; Xiao, K. COVID-19 pneumonia: CT findings of 122 patients and differentiation from influenza pneumonia. Eur. Radiol. 2020, 30, 5463-5469. [CrossRef] [PubMed]

13. Tang, X.; Du, R.H.; Wang, R.; Cao, T.Z.; Guan, L.L.; Yang, C.Q.; Zhu, Q.; Hu, M.; Li, X.Y.; Li, Y.; et al. Comparison of Hospitalized Patients with ARDS Caused by COVID-19 and H1N1. Chest 2020, 158, 195-205. [CrossRef]

14. Cobb, N.L.; Sathe, N.A.; Duan, K.I.; Seitz, K.P.; Thau, M.R.; Sung, C.C.; Morrell, E.D.; Mikacenic, C.; Kim, H.N.; Liles, W.C.; et al. Comparison of Clinical Features and Outcomes in Critically Ill Patients Hospitalized with COVID-19 versus Influenza. Ann. Am. Thorac. Soc. 2020. [CrossRef] [PubMed] 
15. Zayet, S.; Kadiane-Oussou, N.J.; Lepiller, Q.; Zahra, H.; Royer, P.Y.; Toko, L.; Gendrin, V.; Klopfenstein, T. Clinical features of COVID-19 and influenza: A comparative study on Nord Franche-Comte cluster. Microbes Infect. 2020, 22, 481-488. [CrossRef]

16. Wang, H.; Wei, R.; Rao, G.; Zhu, J.; Song, B. Characteristic CT findings distinguishing 2019 novel coronavirus disease (COVID-19) from influenza pneumonia. Eur. Radiol. 2020, 30, 4910-4917. [CrossRef]

17. Lin, L.; Fu, G.; Chen, S.; Tao, J.; Qian, A.; Yang, Y.; Wang, M. CT Manifestations of Coronavirus Disease (COVID-19) Pneumonia and Influenza Virus Pneumonia: A Comparative Study. Am. J. Roentgenol. 2020, 216, 71-79. [CrossRef]

18. Auvinen, R.; Nohynek, H.; Syrjänen, R.; Ollgren, J.; Kerttula, T.; Mäntylä, J.; Ikonen, N.; Loginov, R.; Haveri, A.; Kurkela, S.; et al. Comparison of the clinical characteristics and outcomes of hospitalized adult COVID-19 and influenza patients-A prospective observational study. Infect. Dis. 2020, 53, 111-121. [CrossRef]

19. Yin, Z.; Kang, Z.; Yang, D.; Ding, S.; Luo, H.; Xiao, E. A Comparison of Clinical and Chest CT Findings in Patients with Influenza A (H1N1) Virus Infection and Coronavirus Disease (COVID-19). Am. J. Roentgenol. 2020, 215, 1065-1071. [CrossRef]

20. Lin, Y.H.; Luo, W.; Wu, D.H.; Lu, F.; Hu, S.X.; Yao, X.Y.; Wang, Z.X.; Shi, Y.H. Comparison of clinical, laboratory, and radiological characteristics between SARS-CoV-2 infection and community-acquired pneumonia caused by influenza virus: A cross-sectional retrospective study. Medicine 2020, 99, e23064. [CrossRef]

21. Lee, J.; Lee, Y.H.; Chang, H.H.; Choi, S.H.; Seo, H.; Yoo, S.S.; Lee, S.Y.; Cha, S.I.; Park, J.Y.; Kim, C.H. Comparison of Short-Term Mortality between Mechanically Ventilated Patients with COVID-19 and Influenza in a Setting of Sustainable Healthcare System. J. Infect. 2020, 81, e76-e78. [CrossRef]

22. Kumlin, U.; Olofsson, S.; Dimock, K.; Arnberg, N. Sialic acid tissue distribution and influenza virus tropism. Influenza Other Respir. Viruses 2008, 2, 147-154. [CrossRef] [PubMed]

23. Hamming, I.; Timens, W.; Bulthuis, M.; Lely, A.; Navis, G.; van Goor, H. Tissue distribution of ACE2 protein, the functional receptor for SARS coronavirus. A first step in understanding SARS pathogenesis. J. Pathol. 2004, 203, 631-637. [CrossRef]

24. Rabi, F.A.; Al Zoubi, M.S.; Kasasbeh, G.A.; Salameh, D.M.; Al-Nasser, A.D. SARS-CoV-2 and Coronavirus Disease 2019: What We Know So Far. Pathogens 2020, 9, 231. [CrossRef]

25. Xu, H.; Zhong, L.; Deng, J.; Peng, J.; Dan, H.; Zeng, X.; Li, T.; Chen, Q. High expression of ACE2 receptor of 2019-nCoV on the epithelial cells of oral mucosa. Int. J. Oral Sci. 2020, 12, 8. [CrossRef]

26. Klopfenstein, T.; Zahra, H.; Kadiane-Oussou, N.J.; Lepiller, Q.; Royer, P.-Y.; Toko, L.; Gendrin, V.; Zayet, S. New loss of smell and taste: Uncommon symptoms in COVID-19 patients on Nord Franche-Comte cluster, France. Int. J. Infect. Dis. 2020, 100, 117-122. [CrossRef]

27. Zayet, S.; Klopfenstein, T.; Mercier, J.; Kadiane-Oussou, N.J.; Lan Cheong Wah, L.; Royer, P.-Y.; Toko, L.; Gendrin, V. Contribution of anosmia and dysgeusia for diagnostic of COVID-19 in outpatients. Infection 2020. [CrossRef]

28. Ji, Y.; Ma, Z.; Peppelenbosch, M.P.; Pan, Q. Potential association between COVID-19 mortality and health-care resource availability. Lancet Glob. Health 2020, 8, e480. [CrossRef]

29. Słomka, A.; Kowalewski, M.; Żekanowska, E. Coronavirus Disease 2019 (COVID-19): A Short Review on Hematological Manifestations. Pathogens 2020, 9, 493. [CrossRef]

30. Fathi, N.; Rezaei, N. Lymphopenia in COVID-19: Therapeutic opportunities. Cell Biol. Int. 2020, 44, 1792-1797. [CrossRef] [PubMed]

31. Roberts, N.J. Diverse and Unexpected Roles of Human Monocytes/Macrophages in the Immune Response to Influenza Virus. Viruses 2020, 12, 379. [CrossRef]

32. Xu, B.; Fan, C.; Wang, A.; Zou, Y.; Yu, Y.; He, C.; Xia, W.; Zhang, J.; Miao, Q. Suppressed T cell-mediated immunity in patients with COVID-19: A clinical retrospective study in Wuhan, China. J. Infect. 2020, 81, e51-e60. [CrossRef]

33. Chowdhury, M.A.; Hossain, N.; Kashem, M.A.; Shahid, M.A.; Alam, A. Immune response in COVID-19: A review. J. Infect. Public Health 2020, 13, 1619-1629. [CrossRef]

34. Bonam, S.R.; Kaveri, S.V.; Sakuntabhai, A.; Gilardin, L.; Bayry, J. Adjunct Immunotherapies for the Management of Severely Ill COVID-19 Patients. Cell Rep. Med. 2020, 1, 100016. Available online: https://www.cell.com/cell-reports-medicine/abstract/S266 6-3791(20)30021-5 (accessed on 2 March 2021). [CrossRef]

35. Cohen, S.L.; Gianos, E.; Barish, M.A.; Chatterjee, S.; Kohn, N.; Lesser, M.; Giannis, D.; Coppa, K.; Hirsch, J.; McGinn, T.; et al. Prevalence and Predictors of Venous Thromboembolism or Mortality in Hospitalized COVID-19 Patients. Thromb. Haemost. 2021. [CrossRef] [PubMed]

36. Bai, H.X.; Hsieh, B.; Xiong, Z.; Halsey, K.; Choi, J.W.; Tran, T.M.L.; Pan, I.; Shi, L.-B.; Wang, D.-C.; Mei, J.; et al. Performance of Radiologists in Differentiating COVID-19 from Non-COVID-19 Viral Pneumonia at Chest CT. Radiology 2020, 296, E46-E54. [CrossRef]

37. Zayet, S.; Klopfenstein, T.; Mezher, C.; Gendrin, V.; Conrozier, T.; Ben Abdallah, Y. Coronavirus disease 2019 with spontaneous pneumothorax, pneumomediastinum and subcutaneous emphysema, France. New Microbes New Infect. 2020, $38,100785$. [CrossRef]

38. Shim, S.S.; Kim, Y.; Ryu, Y.J. Novel influenza A (H1N1) infection: Chest CT findings from 21 cases in Seoul, Korea. Clin. Radiol. 2011, 66, 118-124. [CrossRef] 